\title{
PREDICTION OF THE BOUNDARY STATES FOR THIN-WALLED AXISYMMETRIC SHELLS UNDER INTERNAL PRESSURE AND TENSION LOADS
}

\author{
KOZBUR Halyna ${ }^{1}$, SHKODZINSKY Oleh ${ }^{2}$, KOZBUR Ihor ${ }^{3}$, GASHCHYN Nadiia ${ }^{4}$ \\ ${ }^{1}$ Computer Science Department, Ternopil Ivan Puluj National Technical University, Ruska str., 56, Ternopil, \\ Ukraine, kozbur.galina@gmail.com \\ ${ }^{2}$ Automation of Technological Processes and Production Department, Ternopil Ivan Puluj National Technical \\ University, Ruska str., 56, Ternopil, Ukraine, shk@tu.edu.te.ua \\ ${ }^{3}$ Automation of Technological Processes and Production Department, Ternopil Ivan Puluj National Technical \\ University, Ruska str., 56, Ternopil, Ukraine, Kozbur@tu.edu.te.ua \\ ${ }^{4}$ Information Science and Mathematical Modeling Department, Ternopil Ivan Puluj National Technical \\ University, Ruska str., 56, Ternopil, Ukraine, gashchyn.nadia@gmail.com
}

\begin{abstract}
A method for calculating the ultimate true stresses arising in the walls of shells of revolution in the area of uniform plastic deformation is developed in the research. In order to derive the stability loss for the plastic deformation process the criterion of maximum load is taken as the basis, simple differential equations were solved. It has been shown analytically that the level of the boundary true stresses is much lower when the values of the principal stress ratios approach to 2 or $1 / 2$ compared to the adjacent stress states.
\end{abstract}

KEYWORDS: large plastic deformations, true stresses, axisymmetric shells, complex stress state.

\section{Introduction}

The increasing requirements for the load-bearing capacity of shell-type containers, and at the same time the complexity of carrying out the experiments dealing with the loading of axisymmetric thin-walled shells of arbitrary geometry, caused the need of the theoretical apparatus improvement. The load on the axisymmetric shell with uniformly distributed internal pressure and axial tensile force is one of the types of non-momentary loading, with the highest structural strength. Typically, sections of thin-walled spheres, cylinders and cones are used in practice. More complex forms have been studied and, accordingly, used less. Improving the efficiency of thin-walled shells of revolution and, at the same time, the complexity of carrying out experiments on the loading of axisymmetric thin-walled shells of arbitrary geometry are related to the improvement of their calculation methods. The purpose of the paper was to improve methodology of theoretical estimation the safety level of thinwalled vessels subjected to plastic or elastic-plastic deformation under complex stress state and to trace the influence of the stress state and geometric characteristics of the shells on the strength indices.

\section{Brief literature review and problem statement}

The vast majority of studies in the public literature is devoted to the issues of the stability of cylindrical shells, which together with conical and spherical shells are the basic cases of the axisymmetric shell theory [1-5]. The basic theoretical provisions and formulas for determining the stresses in the walls of loaded axisymmetric shells are given in [6]. Authors Middleton J., Owen DR., Blachut J., Zhu L., Boyle J. T., Carbonari, R. C., and others have proposed approaches to the selection and optimization of profiles of thin-walled axisymmetric 
shells loaded with internal pressure with additional loading conditions have been proposed [7-10]. The authors note the complexity of estimating the limit loads in the design of axisymmetric shells. The question of the influence of geometric characteristics of thin-walled shells of other types on their strength characteristics, in particular the value of true stresses at plastic deformation before the moment of localization of deformations, has not been sufficiently investigated. This publication is devoted to the study of the true stress-strain state of thin-walled axisymmetric shells of a positive Gaussian curvature at the stage of plastic deformation stability loss. The results obtained can be used as a basis for predicting the onset of critical states of cylinders and chemical apparatus under pressure.

\section{Objectives of research}

In connection with the above main objectives of the research were: to develop a method of obtaining analytical conditions for the occurrence of boundary states of thin-walled axisymmetric shells under the action of internal pressure and axial tensile for the area of large plastic deformation; to trace the influence of the type of stress state and geometrical characteristics of the axisymmetric shells on the values of true stresses that occur in the shell walls in the area of uniform plastic deformation before the localization of deformations; to obtain results that will contribute to a better understanding of the role of geometric characteristics in ensuring the strength of axisymmetric shells under complex stress state.

\section{$4 \quad$ Obtaining boundary strength conditions}

The axisymmetric shell with thickness $h$ and closed bottoms, the median surface of which is formed by the smooth curve rotation, is uniformly loaded by internal pressure $q$ and the tensile force $N$ in such a way that the formed meridional, circular and radial stresses are principal. The axial stresses are directed along the tangent to the meridian at each point of application (Fig. 1). Large plastic deformations of the shell caused by the action of axisymmetric loading due to internal pressure and tension are considered. The deformations of elastic section are neglected. Kirchhoff's hypotheses about the normality of the elements with respect to the median surface before and after loading, and about the equality of zero stresses normal to the median surface [11], as well as the conditions for the existence of a momentless stress state are accepted. Residual plasticity arising in the direction normal to the middle surface is taken into account. The shell material is assumed to be isotropic and incompressible.

\subsection{Main equations}

The shell element, distinguished by two sufficiently close axial cross sections taken at angle $d \varphi_{1}$ two orthogonal cross sections at the central angle $d \varphi_{2}$, is considered. The edge lengths along the median radii in the circular and meridional directions are denoted respectively by $d s_{1}$ and $d s_{2}$ (Fig. 2). For the element taken at the distance sufficient to neglect the edge effect from the bottoms, the following notation are introduced: $\rho_{m}$ is the current radius of the element median surface (meridian radius), $\rho_{t}$ is the current surface radius the orthogonal to the meridian (circular or latitudinal radius), $\sigma_{m}$ and $\sigma_{t}$ are meridian and circular normal stresses. Radial stress $\sigma_{r}$ is neglected. 


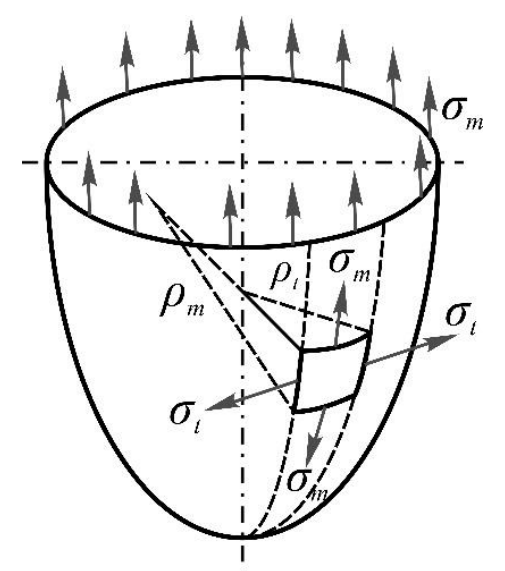

Fig. 1 Axisymmetric shell under complex stress state

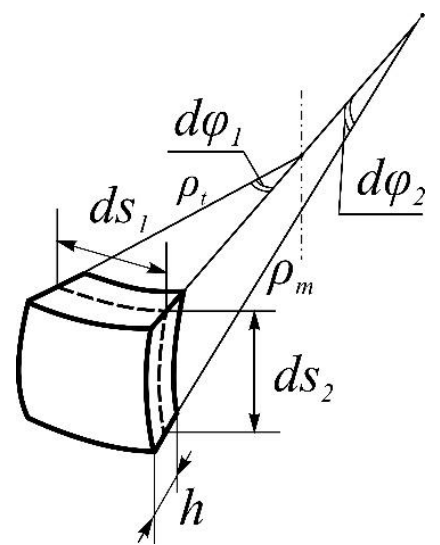

Fig. 2 The axisymmetric shell element

Having projected all the efforts onto the normal to the shell element we get the shell element equilibrium equation:

$$
2 \sigma_{m} h \sin \frac{d \varphi_{2}}{2} d s_{1}+2 \sigma_{t} h \sin \frac{d \varphi_{1}}{2} d s_{2}-q \frac{\rho_{t}-\frac{h}{2}}{\rho_{t}} \frac{\rho_{m}-\frac{h}{2}}{\rho_{m}} d s_{1} d s_{2}=0 .
$$

Taking into account the angles $d \varphi_{1}$ and $d \varphi_{2}$, the equivalence of the small $\sin \left(d \varphi_{1} / 2\right)$ and $d \varphi_{1} / 2, \sin \left(d \varphi_{2} / 2\right)$ and $d \varphi_{2} / 2$, approximations $d \varphi_{1} \approx d s_{1} / \rho_{t}, d \varphi_{2} \approx d s_{2} / \rho_{m}$, the equation (1) is represented in the following way:

$$
\sigma_{m} h d s_{1} \frac{d s_{2}}{\rho_{m}}+\sigma_{t} h \frac{d s_{1}}{\rho_{t}} d s_{2}-q\left(1-\frac{h}{2 \rho_{t}}\right)\left(1-\frac{h}{2 \rho_{m}}\right) d s_{1} d s_{2}=0
$$

Since $h d s_{1} d s_{2} \neq 0$, we get:

$$
\frac{\sigma_{m}}{\rho_{m}}+\frac{\sigma_{t}}{\rho_{t}}=\frac{q}{h}\left(1-\frac{h}{2 \rho_{t}}\right)\left(1-\frac{h}{2 \rho_{m}}\right) .
$$

Accepting $h / \rho_{t} \approx 0, h / \rho_{m} \approx 0$, from (3) we obtain Laplace equation (4).

$$
\frac{\sigma_{m}}{\rho_{m}}+\frac{\sigma_{t}}{\rho_{t}}=\frac{q}{h}
$$

For the cylindrical tube $\left(\rho_{m} \rightarrow \infty\right)$ we obtain the equation of the mean Nadai diameter [10].

Another condition of equilibrium for determining the stresses $\sigma_{m}$ and $\sigma_{t}$ is obtained by considering the equilibrium of the shell part cut off by circular conical surface with generating normal to the middle shell surface at each point of their intersection and inclined to the shell axis at angle $\theta$ (Fig. 3). 
Suppose that only internal pressure $q$ is applied to the shell. Let $O T=\rho_{t}$, $T F=h / 2, T M=r$.

Taking into account that $T M=\rho_{t} \sin \theta$, $K M=T M-K T=\rho_{t} \sin \theta-h /(2 \sin \theta)$, we get $q \pi \cdot K M^{2}=2 \pi h \cdot T M \cdot \sigma_{m}^{q} \sin \theta$, or finally

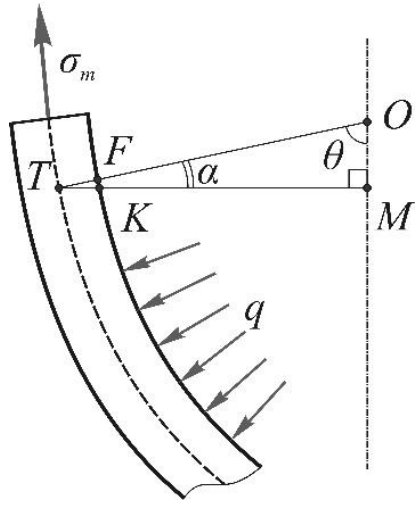

Fig. 3 Fragment of the shell axial section

$$
q \pi\left(\rho_{t} \sin \theta-\frac{h}{2 \sin \theta}\right)^{2}=\sigma_{m}{ }^{q} \cdot 2 \pi h \rho_{t} \sin ^{2} \theta \text {. }
$$

Using the formula $2 \sin ^{2} \theta=1-\cos 2 \theta$ and introducing notation

$$
h / \rho_{t}=\eta
$$

we get

$$
\sigma_{m}{ }^{q}=\frac{q\left(1-\frac{\eta}{1-\cos 2 \theta}\right)^{2}}{2 \eta} .
$$

According to the principle of the forces action independence, the resulting meridian stresses are $\sigma_{m}=\sigma_{m}^{q}+\sigma_{m}^{N}$. Considering (5), we derive:

$$
\sigma_{m}=\frac{q \rho_{t}\left(1-\frac{h}{\rho_{t}(1-\cos 2 \theta)}\right)^{2}}{2 h}+\frac{N}{\pi \rho_{t} h(1-\cos 2 \theta)} .
$$

Taking into account $\alpha=\pi / 2-\theta, \rho_{t} \cos \alpha=r$ and neglecting the correction for the wall thickness in the first summand, we gain the known dependences for meridian stresses in the thin-walled axisymmetric shell by means of momentless theory [13].

From the equation system (3), (8) let us express $q$ and $N$. Let us accept notation

$$
\sigma_{m} / \sigma_{t}=k
$$

Consider the case where the load is followed by significant relative change in sample sizes. Let us denote the conditional large residual deformations in the following way: $\varepsilon_{t}$ - circle, $\varepsilon_{r}$ - radial, $\varepsilon_{m}$ - meridian and take them into account for determination the true stresses, as it is in [14]. Let us introduce the notation $v=\rho_{t} / \rho_{m}$, after transformations we get: 


$$
q=\sigma_{t} \eta \frac{k v \frac{1+\varepsilon_{r}}{1+\varepsilon_{m}}+\frac{1+\varepsilon_{r}}{1+\varepsilon_{t}}}{\left(1-\frac{\eta}{2} \frac{1+\varepsilon_{r}}{1+\varepsilon_{t}}\right)\left(1-\frac{\eta v}{2} \frac{1+\varepsilon_{r}}{1+\varepsilon_{m}}\right)}
$$

and

$$
N=\pi \rho_{t} h \sigma_{m}(1-\cos 2 \theta)\left(1+\varepsilon_{r}\right)\left(1+\varepsilon_{t}\right)\left[1-\frac{\left(1-\frac{\eta}{1-\cos 2 \theta} \frac{1+\varepsilon_{r}}{1+\varepsilon_{t}}\right)^{2}}{2 k\left(1-\frac{\eta}{2} \frac{1+\varepsilon_{r}}{1+\varepsilon_{t}}\right)} \cdot \frac{1+k v \frac{1+\varepsilon_{t}}{1+\varepsilon_{m}}}{1-\frac{\eta v}{2} \frac{1+\varepsilon_{r}}{1+\varepsilon_{m}}}\right] .
$$

Let us use the condition of material incompressibility for the large residual deformations area in the following form

$$
\left(1+\varepsilon_{r}\right)\left(1+\varepsilon_{t}\right)\left(1+\varepsilon_{m}\right)=1
$$

In order to derive the stability loss for the plastic deformation process the criterion of maximum Dorn-Nadai load is taken as the basis $[12,15,16]$. We use conditions $d q=0$ and $d N=0$, in the same way as in [17-19].

\subsection{Boundary true circular stresses and strains}

Let us use the condition

$$
d q=0
$$

We assume that under the internal pressure loading of the shell pinched on the edges, the residual deformations are close to zero: $\varepsilon_{m} \approx 0$ In this case from the incompressibility condition (12) we define

$$
1+\varepsilon_{r}=1 /\left(1+\varepsilon_{t}\right)
$$

Taking into account the accepted assumption, formula (10) is presented as follows

$$
q=\sigma_{t} \eta \frac{\left(1+\varepsilon_{t}\right)\left(k v\left(1+\varepsilon_{t}\right)+1\right)}{\left(\left(1+\varepsilon_{t}\right)^{2}-\eta / 2\right)\left(1+\varepsilon_{t}-\eta v / 2\right)}
$$

Considering the incompressibility condition (16) and regarding in formula (15) $k, \eta, v$ as constant values, condition $d q=0$ is presented in the following way:

$$
\frac{\partial q}{\partial \sigma_{t}} d \sigma_{t}+\frac{\partial q}{\partial \varepsilon_{t}} d \varepsilon_{t}=0
$$

After finding the partial derivatives and substitution in (16) we get: 


$$
\frac{d \sigma_{t}}{\sigma_{t}}+\left[\frac{\left(1+2 k v\left(1+\varepsilon_{t}\right)\right)}{\left(1+\varepsilon_{t}\right)\left(k v\left(1+\varepsilon_{t}\right)+1\right)}-\frac{\left(3\left(1+\varepsilon_{t}\right)^{2}-\eta v\left(1+\varepsilon_{t}\right)-\eta / 2\right)}{\left(\left(1+\varepsilon_{t}\right)^{2}-\eta / 2\right)\left(1+\varepsilon_{t}-\eta v / 2\right)}\right] d \varepsilon_{t}=0
$$

Considering the assumption $\eta^{2} \approx 0$ acceptable for the thin-walled shell, we obtain the general integral of the differential equation (17):

$$
\sigma_{t}=C_{t} \frac{\left[\left(1+\varepsilon_{t}\right)^{2}-\eta / 2\right]^{\frac{4-\left(1-k+2 k^{2}\right) \eta v^{2}}{4-2\left(1+k^{2}\right) \eta v^{2}}}\left[\frac{1+\varepsilon_{t}-\sqrt{\eta / 2}}{1+\varepsilon_{t}+\sqrt{\eta / 2}}\right]^{\frac{v \sqrt{\eta / 2}}{2-\left(1+k^{2}\right) \eta v^{2}}}}{\left[1+\varepsilon_{t}-\eta v / 2\right]^{\frac{(1+k) \eta v^{2}}{2-(1-k) \eta v^{2}}}\left[1+k v\left(1+\varepsilon_{t}\right)\right]},
$$

where $C_{t}$ is the integration constant determined by the experiment results.

In case of the cylindrical shell $v=0$ we get

$$
\sigma_{t}=C_{t}\left[\left(1+\varepsilon_{t}\right)^{2}-\eta / 2\right]
$$

At $\eta \approx 0$ condition (19) coincides with the result obtained in [19].

From (17) we obtain an expression for finding the tangent modulus of the real deformation curve at the moment of loss of stability of the plastic deformation process and the beginning of deformation localization:

$$
\frac{d \sigma_{t}}{d \varepsilon_{t}}=\left[\frac{3\left(1+\varepsilon_{t}\right)^{2}-\eta v\left(1+\varepsilon_{t}\right)-\eta / 2}{\left(\left(1+\varepsilon_{t}\right)^{2}-\eta / 2\right)\left(1+\varepsilon_{t}-\eta v / 2\right)}-\frac{1+2 k v\left(1+\varepsilon_{t}\right)}{\left(1+\varepsilon_{t}\right)\left(k v\left(1+\varepsilon_{t}\right)+1\right)}\right] \sigma_{t} .
$$

Considering the partial load case of a cylindrical tube $(v=0)$ and neglecting the subtlety index $(\eta \approx 0)$, we obtain the following expression for finding the tangent module

$$
\frac{d \sigma_{t}}{d \varepsilon_{t}}=\frac{2}{1+\varepsilon_{t}} \sigma_{t}
$$

\subsection{Boundary true circular stresses and strains}

Let us use the condition

$$
d N=0
$$

Suppose that in the area of uniform plastic deformations the shell convexity changes and the thinness index remains practically unchanged, i.e. $\eta\left(1+\varepsilon_{r}\right) /\left(1+\varepsilon_{t}\right) \approx \eta$. Then $(10)$ is as follows

$$
N=\pi \rho_{t} h \sigma_{m}(1-\cos 2 \theta)\left(1+\varepsilon_{r}\right)\left(1+\varepsilon_{t}\right)\left[1-\frac{\left(1-\frac{\eta}{1-\cos 2 \theta}\right)^{2}}{2 k\left(1-\frac{\eta}{2}\right)} \cdot \frac{1+k v \frac{1+\varepsilon_{t}}{1+\varepsilon_{m}}}{1-\frac{\eta v}{2} \frac{1+\varepsilon_{r}}{1+\varepsilon_{m}}}\right] .
$$


Taking into account (9) and Poisson coefficient $\mu=0,5$ we get

$$
\varepsilon_{m} / \varepsilon_{t}=n
$$

where

$$
n=(2 k-1) /(2-k) \text {. }
$$

Considering the incompressibility condition (12) for large deformation area in the form $\left(1+\varepsilon_{r}\right)\left(1+\varepsilon_{t}\right)=1 /\left(1+\varepsilon_{m}\right)$ and formulas (9), (24), from formula (23) we get

$$
N=\pi \rho_{t} h(1-\cos 2 \theta) \frac{\sigma_{m}}{1+\varepsilon_{m}}\left[1-\frac{\left(1-\frac{\eta}{1-\cos 2 \theta}\right)^{2}}{2 k\left(1-\frac{\eta}{2}\right)} \cdot \frac{1+\varepsilon_{m}+k v\left(1+\frac{1}{n} \varepsilon_{m}\right)}{1+\varepsilon_{m}-\frac{\eta v}{2}\left(1+\frac{1}{n} \varepsilon_{m}\right)}\right] .
$$

Condition (22) is reduced to the differential equation:

$$
\begin{aligned}
& \frac{d \sigma_{m}}{\sigma_{m}}=\left\{1 /\left(1+\varepsilon_{m}\right)+\right. \\
& \left.+\frac{v a\left(k+\frac{\eta}{2}\right)\left(\frac{1}{n}-1\right)}{\left(1+\varepsilon_{m}-\frac{\eta v}{2}\left(1+\frac{\varepsilon_{m}}{n}\right)\right)\left[2 k\left(1-\frac{\eta}{2}\right)\left(1+\varepsilon_{m}-\frac{\eta v}{2}\left(1+\frac{\varepsilon_{m}}{n}\right)\right)-a\left(1+\varepsilon_{m}+k v\left(1+\frac{\varepsilon_{m}}{n}\right)\right)\right]}\right\} d \varepsilon_{m},
\end{aligned}
$$

where $a=(1-\eta /(1-\cos 2 \theta))^{2}$.

The general equation integral (27) is:

$$
\sigma_{m}=C_{m} \frac{\left(1+\varepsilon_{m}\right)\left[1-\frac{\eta v}{2}+\varepsilon_{m}\left(1-\frac{\eta v}{2 n}\right)\right]}{k\left(1-\frac{\eta}{2}\right)(2-\eta v)-a(1+k v)+\varepsilon_{m}\left[k\left(1-\frac{\eta}{2}\right)\left(2-\frac{\eta v}{n}\right)-a\left(1+\frac{k v}{n}\right)\right]} .
$$

If $v=0$ we have the case of cylindrical tube, then $\sigma_{m}=\sigma_{z}, \varepsilon_{m}=\varepsilon_{z}, C_{m}=C_{z}$. The boundary condition (26) is as follows $\sigma_{z}=\frac{C_{z}\left(1+\varepsilon_{z}\right)}{2 k(1-\eta / 2)-a}$ or, with notation $1 /(2 k(1-\eta / 2)-a)=C_{z}^{*}$

$$
\sigma_{z}=C_{z}^{*}\left(1+\varepsilon_{z}\right)
$$

Condition (29) coincides with condition for thin-walled cylinder, obtained in [19].

From (27) we obtain an expression for finding the tangent modulus, held at the point of a real deformation diagram corresponding to the beginning of the localization of deformations: 


$$
\begin{aligned}
& \frac{d \sigma_{m}}{d \varepsilon_{m}}= \\
& =\left\{\frac{1}{1+\varepsilon_{m}}+\frac{v a\left(k+\frac{\eta}{2}\right)\left(\frac{1}{n}-1\right)}{\left(1+\varepsilon_{m}-\frac{\eta v}{2}\left(1+\frac{\varepsilon_{m}}{n}\right)\right)\left[2 k\left(1-\frac{\eta}{2}\right)\left(1+\varepsilon_{m}-\frac{\eta v}{2}\left(1+\frac{\varepsilon_{m}}{n}\right)\right)-a\left(1+\varepsilon_{m}+k v\left(1+\frac{\varepsilon_{m}}{n}\right)\right)\right]}\right\} \sigma_{m}
\end{aligned}
$$

For the partial case of a cylindrical tube $(v=0)$ we obtain the condition:

$$
\frac{d \sigma_{m}}{d \varepsilon_{m}}=\frac{1}{1+\varepsilon_{m}} \sigma_{m}
$$

obtained by A. Nadai for the case of uniaxial tensile [12]. The second addition to the right of formula (30) corrects the slope of the normal to the plot relative to the axis of the shell, the type of stress state and geometry $v, \eta$. Dependences (21) and (31) show that the tangent of the slope of the tangent to the true deformation curve, held at a point that reflects the moment of onset of deformation localization from the action of internal pressure, is twice the value of the tangent modulus for uniaxial tensile.

\subsection{Constants for conditions (18) and (28)}

In order to find $C_{t}$ in general integral (18) we consider the case $k=0,5$. The boundary circular stresses and strains $\varepsilon_{t}^{b}, \sigma_{t}^{b}$ correspond to the sample fracture moment for brittle material state and the beginning of deformations localization for plastic material state (Table 1). The experimental results for cylindrical thin-walled samples that have been made of grades of structural alloyed steel used for the manufacture of pressure vessels are given in [14], [19].

Table 1 Calculation of integration constant values for condition (18)

\begin{tabular}{|l|c|c|c|c|c|}
\hline \multicolumn{1}{|c|}{ Material grade } & $\eta=h / \rho_{t}$ & $\varepsilon_{t}^{b}$ & $\sigma_{t}^{b}$ & $C_{t}$ & $\begin{array}{c}\text { Reduced tension } \\
\sigma_{t}^{b} / C_{t}\end{array}$ \\
\hline Steel 10MnH2MoV & $0,06^{*}$ & 0,036 & $705 \mathrm{MPa}$ & 675,74 & 1,04 \\
\hline Steel 15Cr2MoV & 0,067 & 0,021 & $746 \mathrm{MPa}$ & 739,39 & 1,01 \\
\hline Steel 15Cr2HMoV & 0,067 & 0,024 & $745 \mathrm{MPa}$ & 733,94 & 1,02 \\
\hline
\end{tabular}

*the average value for thin-walled shells with $\eta=0,04 . .0,08$ is taken

Based on the boundary values $\sigma_{m}=\sigma_{m}^{b}, \varepsilon_{m}=\varepsilon_{m}^{b}$ of cylindrical tubes for $k=2$ integration steels $C_{m}$ are determined for condition (28). The results are shown in Table 2.

Table 2 Calculation of integration constant values for condition (28)

\begin{tabular}{|l|c|c|c|c|c|}
\hline \multicolumn{1}{|c|}{ Material grade } & $\eta=h / \rho_{t}$ & $\varepsilon_{m}^{b}$ & $\sigma_{m}^{b}$ & $C_{m}$ & $\begin{array}{c}\text { Reduced tension } \\
\sigma_{m}^{b} / C_{m}\end{array}$ \\
\hline Steel 10MnH2MoV & $0,06^{*}$ & 0,043 & $722 \mathrm{MPa}$ & 692,23 & 1,04 \\
\hline Steel 15Cr2MoV & 0,067 & 0,042 & $830 \mathrm{MPa}$ & 796,55 & 1,04 \\
\hline Steel $15 \mathrm{Cr} 2 \mathrm{HMoV}$ & 0,067 & 0,030 & $800 \mathrm{MPa}$ & 776,70 & 1,03 \\
\hline
\end{tabular}

*the average value for thin-walled shells with $\eta=0,04 . .0,08$ is taken 


\subsection{Graphical analysis of conditions (18) and (28)}

The graphs of the strength loss conditions (18) for the case $\sigma_{t}>\sigma_{m}$ were constructed for $\eta=0,06$ (Fig. 4, a). The deformations values $\varepsilon_{t}^{b}$ and reduced stresses $\sigma_{t}^{b} / C_{t}$ were selected according to Table 1 .

The graphs of condition (28) for the case $\sigma_{m}>\sigma_{t}$ are shown in Fig. 4, b. The deformation values range $\varepsilon_{m}^{b}$, reduced stresses $\sigma_{m}^{b} / C_{m}$ and value $\eta=0,06$ were selected according to the data in Table 2.

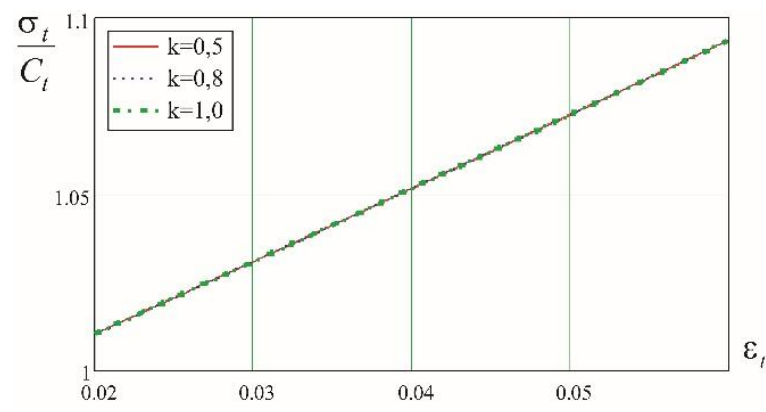

a)

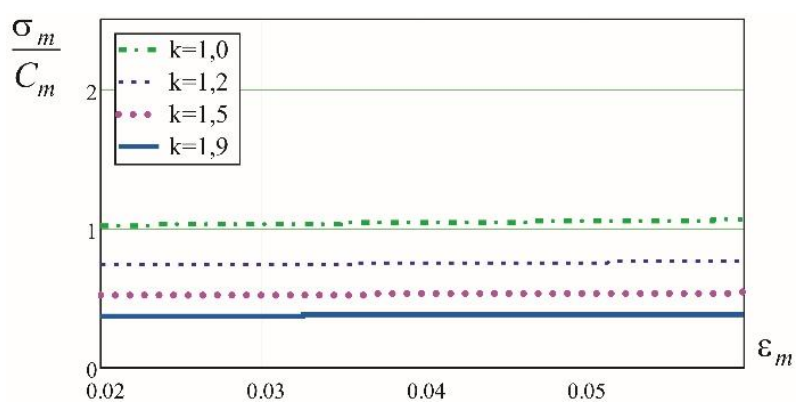

b)

Fig. 4 Graphic images: a) condition (18) for $\sigma_{t}>\sigma_{m}$; b) condition (28) for $\sigma_{m}>\sigma_{t}$

The analysis of the graph of condition (18), made for $\sigma_{t}>\sigma_{m}>0$ (Fig. 4, a), showed that for complex stress states close to $k=0,5$, the reduced limit true stresses practically do not depend on the type of stress state, but increase in proportion with the residual deformations.

On the contrary, analysis of the graphs constructed for (28) at $\sigma_{m}>\sigma_{t}>0$ (Fig. 4, b) showed a significant decrease in the shear strength resource when $k \rightarrow 2$ compared to adjacent stress states. As the level of residual deformation increases in the meridional direction, the corresponding limit true stresses increase, but not rapidly.

The boundary curves described by condition (18) of the principal stress ratios $k=0,5$ for cylindrical tube $(v=0)$ are shown in Fig. 5. Analysis of Fig. 5 showed a decrease in the level of the boundary true stresses of a cylindrical tube in a circular direction with increasing values $\eta$.

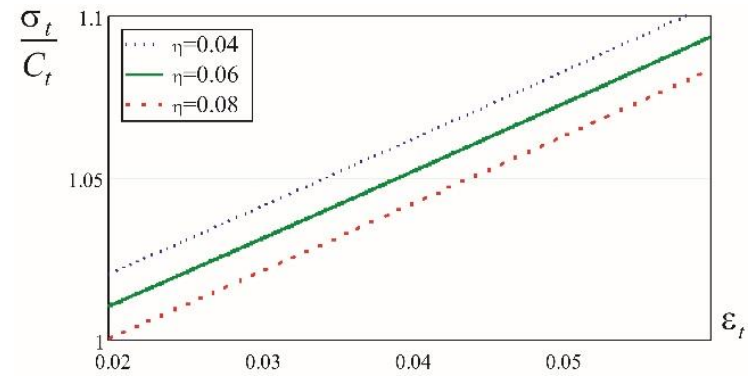

Fig. 5 Graphical representation of dependence (18) of the boundary circular stresses $\sigma_{t}$ and residual deformations $\varepsilon_{t}$ on parameter $\eta$
A certain level of boundary stresses is achieved at a higher level of plastic deformation if the ratio of the initial thickness of the wall of the tube to its initial radius is greater, respectively. For other values $k=0,5 \ldots 1$ the qualitative and quantitative pattern remained practically unchanged.

The graphs of conditions (28) shown in Fig. 6 were constructed for different $k$. The case of cylindrical tube $(v=0)$ was considered. For other $v$ and $k$ the qualitative pattern did not change. 


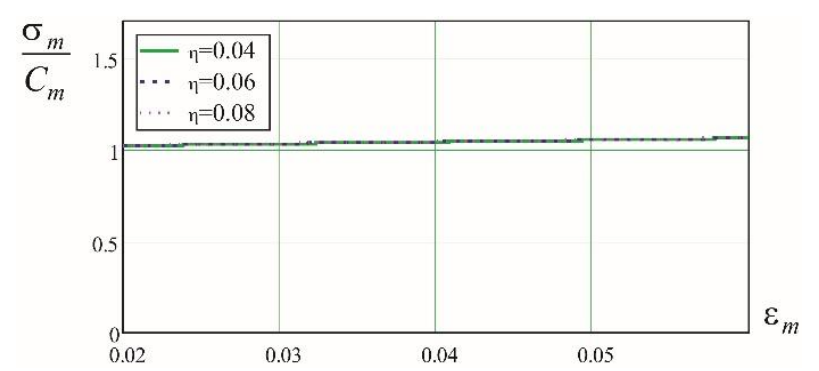

a) $k=1$

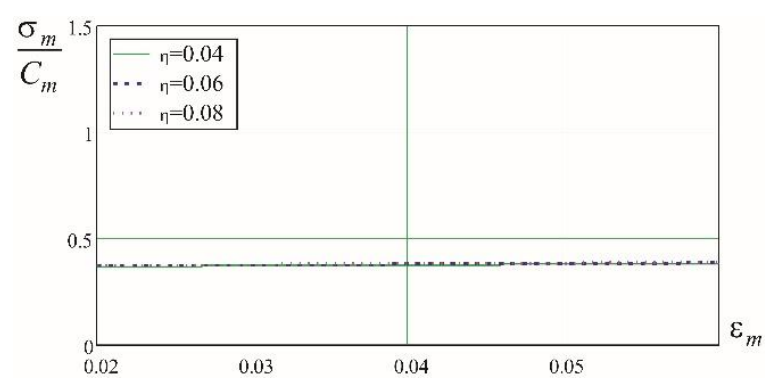

b) $k=1,9$

Fig. 6 Graphical representation of dependence (28) of the boundary meridian stresses $\sigma_{m}$ and residual deformations $\varepsilon_{m}$ on parameter $\eta$ at different $k$

Analysis of the graphs in Fig. 6 showed the decrease in the values $\sigma_{m}$ at $k \rightarrow 2$, the influence $\eta$ on the boundary stresses $\sigma_{m}$ value practically is not observed.

The family of boundary curves (18) for different parameter $v$ values is shown in Fig. 7. The thinness index is accepted to be $\eta=0,06$.

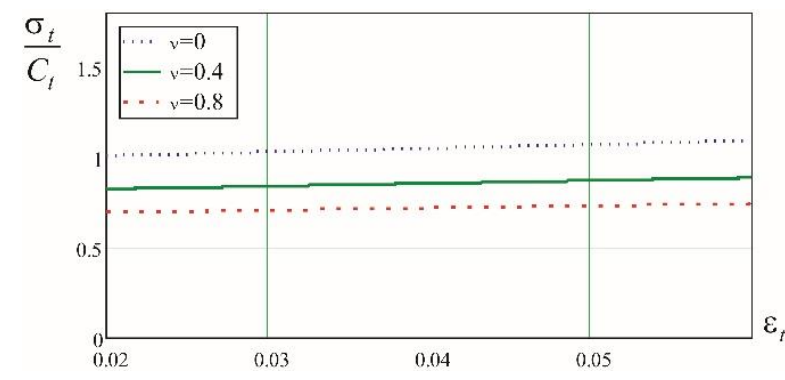

a) $k=0,5$

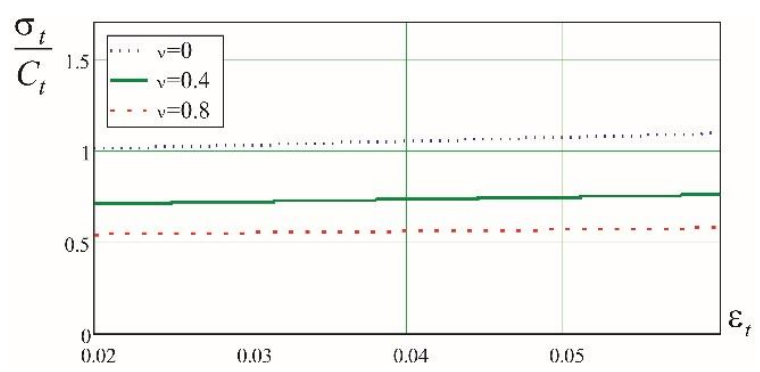

b) $k=1,0$

Fig. 7 Graphical representation of dependence (18) of the boundary true circular stresses $\sigma_{t}$ and residual deformations $\varepsilon_{t}$ on parameter $v$

The graphs of the dependences (18) showed that under the action of internal pressure only (Fig. 7, a), the boundary true stresses in the circular direction decrease as the convex value increases. In the conditions of simultaneous action of internal pressure and tensile force, the boundary circular stresses with the same convexity indices decrease somewhat (Fig. 7,b). When $k \rightarrow 1$, the effect of the convexity of the shell on its strength increases.

The boundary curves obtained from (28) at $\eta=0,06$ are shown in Fig. 8.

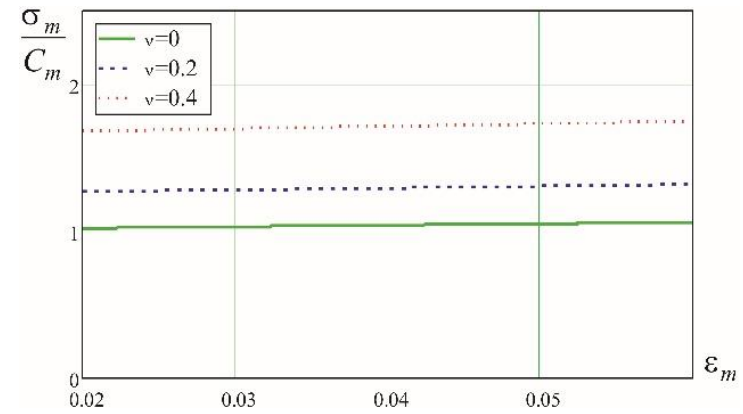

a) $k=1$

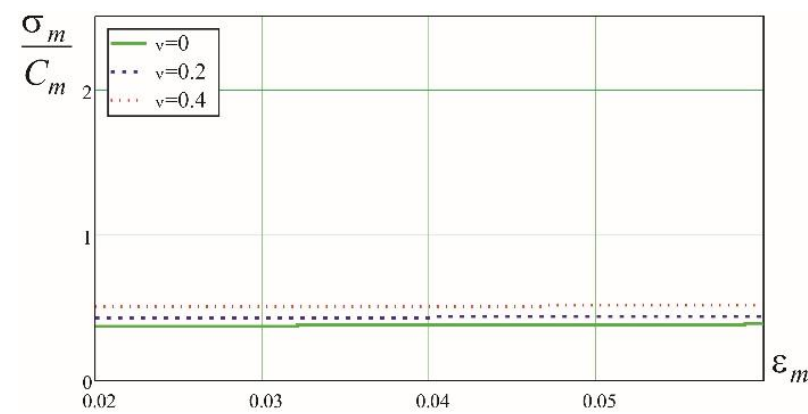

b) $k=1,9$

Fig. 8 Graphical representation of dependence (28) of the boundary true meridian stresses $\sigma_{m}$ and residual deformations $\varepsilon_{m}$ on parameter $v$ 
Analysis of graphs in Fig. 8 showed that with the increase of the convexity of the shell from $v=0$ (cylindrical tube) to $v=1$ (spherical shell) the level of the boundary true stresses in the meridional direction increases, that is, the walls of the shell before the localization of deformations develop larger residual deformations in the corresponding direction. There is an increased effect of the convexity of the shell at $k \rightarrow 1$. When $k \rightarrow 2$, the level of the ultimate true meridional stresses decreases, the effect of the convexity of the shell is almost neutralized.

\section{$5 \quad$ Results and discussion}

Analysis of the boundary strength conditions obtained in the article showed that the critical load value, which causes the occurrence of plastic deformations in the walls of thin-walled axisymmetric shell, depends on the material physical properties, the stress state type, the geometric shape and dimensions of the shell and is proved by the experiments.

As the values of the principal stress ratios approached 2, a significant decrease in the material strength resource was observed, which is shown by the lower level of the boundary true stresses that develop in the shell walls before the onset of deformation localization. The fact that the calculated strength of the cylindrical tube in a circular direction decreases with increasing values $\eta$ is analytically confirmed.

With only internal pressure, the thin-walled axisymmetric shell increases its strength and durability if the shell shape approaches spherical. In conditions of simultaneous action of internal pressure and tensile axial force, the boundary circular true stresses with the same convexity indices are somewhat reduced compared to the stresses that develop in the walls of the shell only from the action of internal pressure. When the value $k$ approaches one, the influence of the shell convexity on its strength increases.

The results of the verification of the achieved results by means of numerical modeling will be covered by the authors in the following articles.

\section{CONCLUSIONS AND PERSPECTIVES}

The technique of prediction of critical values of stresses in the walls of axisymmetric shells, loaded with internal pressure and tensile forces, is proposed in the article, taking into account the change in the shells geometry. For this purpose, dependences are proposed for the calculation of the limit level of the real stresses formed in the walls of a convex shell due to the action of internal pressure and longitudinal tensile. The obtained dependencies make it possible to construct an algorithm for obtaining the numerical critical values of stresses in thin-walled axisymmetric shells based on the results of uniaxial tensile experiments and predict vessels behavior under load.

The perspectives in this work are: checking the adequacy of the obtained dependencies for a specific class of plastic materials; application of the proposed approach to finding the level of critical loads and residual deformations to shells with an average value of the wall thickness to the diameter of the middle surface, as well as to the axisymmetric shells of negative Gaussian curvature; the use of software to automate calculations and improve their accuracy.

\section{REFERENCES}

[1] Tomita, Y., Shindo, A., Nagai, M. “Axisymmetric deformation of circular elastic-plastic tubes under axial tension and internal pressure", International Journal of Mechanical Sciences 26, pp. 437 - 444, 1984. DOI: 10.1016/0020-7403(84)90033-X 
[2] Shahabi, M., Esmaeilnejad, M., Ghasemi, A. "A thin-walled tube subjected to combined internal pressure and axial load under different loading paths", Strojnícky časopis Journal of Mechanical Engineering 63 (5-6), p. 307, 2012.

[3] Chmelko, V., Krššák, P. "The elasto-plastic state solution of a heavy-wall cylindrical pressure vessel using bilinear stress-strain model. Part 1: Derivation of analytical relations", Strojnícky časopis - Journal of Mechanical Engineering 64 (1), p. 51, 2013.

[4] Bazhenov, V. G., Lomunov, V. K. "An experimental-theoretical study of the process of neck formation under tension of a steel tubular specimen before rupture", In: Problems of durability and plasticity, Publishing House of the UNN, Nizhny Novgorod, Russia, pp. 35 - 41, 2001.

[5] Grigolyuk, E. I., Kabanov, V., "Stability of shells", Science, Moskow, USSR, 1978.

[6] Pisarenko, G.S., Agaev, V.A., Kvitka, A.L., Popkov, V.G., Umanskiy, E.S. "Strength of Materials" $4^{\text {th }}$ ed., Vischa shkola, Kiev, USSR, 1979.

[7] Middleton, J., Owen, DRJ., "Automated design optimization to minimize shearing stress in axisymmetric pressure-vessels", Nuclear Engineering and Design 44 (3), pp. 357 - 366, 1977.

[8] Błachut, J. "Minimum weight of internally pressurised domes subject to plastic load failure", Thin-Walled Structures 27(2), pp. 127 - 146, 1997. DOI:10.1016/s02638231(96)00036-5

[9] Zhu, L., Boyle, J.T., "Optimal shapes for axisymmetric pressure vessels: a brief overview", Journal of Pressure Vessel Technology-transactions of the ASME 122 (4), p. 443, 2000. DOI:10.1115/1.1308572

[10] Carbonari, R. C., Muñoz-Rojas, P. A., Andrade, E. Q., Paulino, G. H., Nishimoto, K., Silva, E. C. N. "Design of pressure vessels using shape optimization: An integrated approach", International Journal of Pressure Vessels and Piping 88 (5-7), pp. 198 - 212, 2011. DOI: 10.1016/j.ijpvp.2011.05.005

[11] "Kirchhoff - Love plate theory". [online] Available at: URL: https://en.wikipedia.org/wiki/Kirchhoff\%E2\%80\%93Love_plate theory.

[12] Nadai, A. "Plasticity and destruction of solids", Volume 1, ed. Shapiro, G. S., in 2 volumes, Foreign Literature, Moscow, USSR, 1954.

[13] "Soprotivlenie materialov [Strength of Materials]", In: Book editor with. Smirnov, A.F., Vysshaya shkola, Moskow, 1975.

[14] Lebedev, A.A., Kovalchuk, B.I., Giginyak, F.G., Lamashevsky, V.P. "Mechanical Properties of Structural Materials under Complex Stress", In: Book editor with Lebedev, A.A., In Yure, Kyiv, Ukraine, 2003. ISBN 966-8088-36-0

[15] Friedman, Ya. B. "Mechanical properties of metals", All-Union order of Lenin scientific research institute of aviation materials, Oborongiz, Moskow, 1946.

[16] Robert, H. "Mathematical theory of plasticity" ed. trans. Grigolyuk, E. I., Gostekhizdat, Moscow, 1956.

[17] Kaminsky, A.A., Bastun, V.N. "Deformation hardening and fracture of metals at variable loading processes", Scientific Thought, Kyiv, 1985.

[18] Shkodzinsky, O.K., Kozbur, G.V. "Investigation of the stability of the process of plastic deformation of a thin-walled tube under conditions of complex stress state", Bulletin of the TDTU 14 (3), pp. $24-31,2009$.

[19] Giginyak, F.F., Lebedev, A.A., Shkodzinsky, O.K. "Strength of structural materials at low cycle load under conditions of complex stress state", Scientific Thought, Kyiv, 2003. ISBN 9660007868 